\title{
Gemcitabine treatment causes resistance and malignancy of pancreatic cancer stem-like cells via induction of IncRNA HOTAIR
}

\author{
LI WANG, PING DONG, WEIGUO WANG, MINGQUAN HUANG and BOLE TIAN
}

\author{
Department of Pancreatic Surgery, West China Hospital, Sichuan University, Chengdu, Sichuan 610064, P.R. China
}

Received January 25, 2017; Accepted July 14, 2017

DOI: $10.3892 / \mathrm{etm} .2017 .5151$

\begin{abstract}
Gemcitabine is the first-line chemotherapeutic agent for advanced adenocarcinoma of the pancreas, despite the high risk of chemoresistance as a major disadvantage. In the past few years, significant advances have been made in the field of pancreatic cancer stem-like cells (CSCs) and their critical roles in drug resistance, invasion and metastasis, which are tightly regulated by long non-coding RNAs (lncRNAs). The present study demonstrated that HOX antisense intergenic RNA (HOTAIR) is not different between the pancreatic cancer cell line PANC-1 and its enriched CSC sub-population. However, after gemcitabine treatment, the expression levels of HOTAIR in CSCs were induced, but not in PANC-1 cells. HOTAIR induced by gemcitabine failed to cause chemoresistance, but promoted the clonogenicity, proliferation and migration of the cells. By introducing HOTAIR using lentivirus, chemoresistance was induced and the self-renewal capacity, proliferation and migration were significantly promoted. By contrast, HOTAIR knockdown in PANC-1 CSCs treated with or without gemcitabine decreased the cell proliferation, altered the cell cycle progression and induced apoptosis, demonstrating its critical roles in regulating the malignant character of PANC-1 CSCs. In conclusion, the present study demonstrated that HOTAIR may be induced by gemcitabine and acts as a tumor promoter by inhibiting the chemosensitivity, and promoting the self-renewal capacity, proliferation and migration of PANC-1 CSCs, which supports its potential application as a novel therapeutic approach for pancreatic cancer.
\end{abstract}

\section{Introduction}

Pancreatic cancer, one of leading causes of cancer-associated mortality in Western countries, has an extremely poor prognosis

Correspondence to: Professor Bole Tian, Department of Pancreatic Surgery, West China Hospital, Sichuan University, 37 Guo Xue Xiang, Chengdu, Sichuan 610064, P.R. China E-mail: tianbole@163.com

Key words: pancreatic cancer, cancer stem-like cells, HOX antisense intergenic RNA, gemcitabine, chemoresistance with an overall five-year survival rate of $<5 \%$ and a median survival of $<1$ year $(1,2)$. The poor prognosis of pancreatic cancer is mainly due to the malignant behavior of pancreatic cancer, including metastasis, recurrence and chemoresistance. As major hallmarks of pancreatic cancer, extensive local invasion, early systemic dissemination and resistance to most cytotoxic drugs also attribute to its malignancy.

At present, the clinical standard of care for early-diagnosed or advanced pancreatic cancer is chemotherapy with 2',2'-difluorodeoxycytidine ( $\mathrm{dFdC}$; gemcitabine), a cytotoxic nucleoside analogue. Gemcitabine has a relatively low tumor response rate of $\sim 15 \%$ and offers a median survival time of 5 months (3), although it only extends survival by a mere 5 weeks on average (4). Of note, pancreatic tumors in a substantial number of patients are already (or rapidly become) chemoresistant to gemcitabine and display a loss of fundamental response (2). Thus, improving chemosensitivity is a strategy for increasing therapeutic effects on pancreatic cancer. For this purpose, recent studies have identified several chemoresistance mechanisms associated with the metabolism and molecular targets of gemcitabine $(5,6)$.

The discovery of cancer stem-like cells (CSCs) has provided novel insight into carcinogenesis and the effects of cancer therapy. It has been suggested that sub-populations of CSCs within solid tumors sustain the formation and growth of the tumor. The presence of CSCs also accounts for tumor recurrence due to their self-renewal capacity and metastatic potential $(7,8)$. Compared with other tumor cells, CSCs also present with a significantly increased chemoresistance to conventional therapeutics, including gemcitabine (9). Studies assessing specific oncogene models of cancer and specific signaling pathways revealed that CSCs tightly mediate chemoresistance (10), which has been demonstrated in various cancer types, including lung (11), pancreatic (12), prostate (13), liver (14) and head and neck squamous cancer (15). Yin et al (16) reported that enrichment of CSCs in the Panc-1 pancreatic cancer cell line increased the migration ability and resistance to gemcitabine, although the mechanism has remained to be elucidated.

Long non-coding RNAs (lncRNAs) are a class of endogenous cellular RNAs of $<200$ nucleotides in length that lack an open reading frame of significant length (17). In recent years, accumulating evidence has indicated regulatory roles of IncRNAs regarding the malignant character of various cancer 
types. Overexpression of metastasis-associated lung adenocarcinoma transcript 1 (MALAT-1), a highly evolutionarily conserved and ubiquitously expressed lncRNA, in pancreatic cancer cells increased the proportion of pancreatic CSCs, maintained their self-renewal capacity, decreased their sensitivity to anticancer drugs and accelerated tumor angiogenesis in vitro (18). HOX transcript antisense RNA (HOTAIR) has been intensely investigated in several cancer types, including lung (19), prostate (20) and pancreatic cancers (21). HOTAIR and MALAT-1 have received increasing attention due to their aberrant expression in cancer tissues (18-21).

In the present study, the CSC sub-population from Panc-1 cells was enriched using serum-free medium and exposed to different concentration of gemcitabine. The cells were then subjected to reverse-transcription quantitative polymerase chain reaction (RT-qPCR) analysis to detect the expression levels of several lncRNAs. Apart from HOTAIR and MALAT-1 which were assessed due to their tight association with the malignancy of pancreatic cancer, maternally expressed 3 (MEG3) (21), protein phosphatase 3 catalytic subunit $\beta$ (PPP3CB), mitogen-activated protein kinase kinase kinase 14 (MAP3K14) and death-associated protein kinase 1 (DAPK1) (22) were also assessed. A significantly higher expression of HOTAIR was observed in Panc-1 and CSCs enriched from Panc-1 after exposure to gemcitabine. Further experiments strongly suggested that HOTAIR may have a role in pancreatic stemness, increasing chemoresistance to gemcitabine, attenuating apoptosis and promoting proliferation. Taken together, these results provided novel insight into the negative effects of gemcitabine exposure on the sub-population of pancreatic CSCs by upregulating HOTAIR and uncovered a role for the lncRNA HOTAIR as a potential stemness regulator and novel therapeutic target.

\section{Materials and methods}

Cell culture and gemcitabine treatment. The Panc-1 pancreatic cancer cell line purchased from American Type Culture Collection (ATCC; Manassas, VA, USA) and cultured in Dulbecco's Modified Eagle's Medium (DMEM; Thermo Fisher Scientific, Inc., Waltham, MA, USA) supplemented with $10 \%$ fetal bovine serum (FBS; Sigma-Aldrich; Merck KGaA, Darmstadt, Germany), penicillin (100 U/ml) and streptomycin $(100 \mathrm{U} / \mathrm{ml}$; Thermo Fisher Scientific, Inc.) at $37^{\circ} \mathrm{C}$ in an incubator with $5 \% \mathrm{CO}_{2}$. To stimulate pancreatic cancer cells to form tumor spheres in suspension, the following culture conditions were used: Panc-1 cells were suspended using Trypsin (Thermo Fisher Scientific, Inc. CA, USA) and diluted to a density of $10^{6}$ cell $/ \mathrm{ml}$ in serum-free medium, which was composed of DMEM/F12 supplemented with 2\% B-27 (Thermo Fisher Scientific, Inc.), $20 \mathrm{ng} / \mathrm{ml}$ epidermal growth factor (EGF) and $10 \mathrm{ng} / \mathrm{ml}$ fibroblast growth factor-basic (bFGF; PeproTech, Rocky Hill, NJ, USA). The cells were passaged every 12 days and replated in the serum-free medium. The spheres forming under these conditions were named PANC-1 CSCs.

For gemcitabine treatment, cells were cultured in SFM supplemented with 25,50 or $100 \mu \mathrm{g} / \mathrm{ml}$ gemcitabine (Sigma-Aldrich; Merck KGaA, Darmstadt, Germany) for 24 h, and then subjected to the other assays.
Construction of lentiviral particles containing HOTAIR coding sequence. The HOTAIR coding sequence was amplified by RT-PCR and then cloned into the pCDH-MSCV-mcs-GFP lentiviral vector (System Biosciences; Palo Alto, CA, USA) at the EcoRI and NotI sites (Fermentas; Thermo Fisher Scientific, Inc., Waltham, MA, USA). For generating lentiviral particles, the packaging vectors psPAX2 and pMD2.G (Addgene, Inc., Cambridge, MA, USA) were co-transfected into 293T cells (ATCC) with pCDH-MSCV-msc-GFP lentiviral vector containing HOTAIR coding sequence. After $72 \mathrm{~h}$, the supernatant was collected and the titer was determined.

Construction of the small hairpin RNA targeting HOTAIR (shHOTAIR) vector and plasmid transfection. For constructing the vector encoding shHOTAIR, the following specific oligonucleotides targeting HOTAIR were synthesized (Shenggong, Shanghai, China): Sense,5'-GATCCGCCACA TGAACGCCCAGAGATTTTCAAGAGAAATCTCTGGGC GTTCATGTGGTTTTTTG-3' and anti-sense,5'-AATTCA AAAAACCACATGAACGCCCAGAGATTTCTCTTGAAA ATCTCTGGGCGTTCATGTGGCG-3'. After annealing, the double-stranded DNA was inserted into pENTR.hU6hH1 empty vector between NdeI and EcoRI sites (Fermentas; Thermo Fisher Scientific, Inc.). The pENTR-shHOTAIR plasmids or empty vectors were transfected into target cells using Lipofectamine 2000 ${ }^{\mathrm{TM}}$ (Thermo Fisher Scientific, Inc.).

$R T-q P C R$. Total RNA extracted from target cells by using TRIzol reagent (Thermo Fisher Scientific, Inc.) was used for complementary (c)DNA synthesis. Briefly, M-MLV first strand cDNA Synthesis kit (Omega Bio-Tek, Inc., Norcross, GA, USA) was used following manufacturer's guide. The sequences of the primers used in the present study were as follows: HOTAIR forward, 5'-GAGAGAGGGAGCCCAGAGTT-3' and reverse, 5'-GCTTGGGTGTAATTGCTGGT-3'; MALAT-1 forward, 5'-TGTGTGCCAATGTTTCGTTT-3' and reverse, 5'-AGG AGAAAGTGCCATGGTTG-3'; MEG3 forward, 5'-TTGACA GGTCAGTCCCTTCC-3' and reverse, 5'-TTCCACGGAGTA GAGCGAGT-3'; PPP3B forward, 5'-CAACCATGAATG CAGACACC-3' and reverse, 5'-TGGTGAAAGTCCACCATG AA-3'; MAP3K14 forward, 5'-CAAGCCTCTGAAGGAACC AG-3' and reverse, 5'-AGGGATGAGGCAGTCTGCTA-3'; DAPK1 forward, 5'-ATGATCCCACGTCAATCCAT-3' and reverse, 5'-CCACCAGGACAACTTGGAGT-3'; GAPDH forward, 5'-GGAGCGAGATCCCTCCAAAAT-3' and reverse, 5'-GGCTGTTGTCATACTTCTCATGG-3'. PCR was performed on an Applied Biosystems 7500 Real-time system (Thermo Fisher Scientific, Inc.) using Power SYBR-Green PCR Master Mix (Thermo Fisher Scientific, Inc.). The thermocycling conditions were as follows: $5 \mathrm{~min}$ at $50^{\circ} \mathrm{C}$ and $5 \mathrm{~min}$ at $95^{\circ} \mathrm{C}$, followed by 35 cycles of $30 \mathrm{sec}$ at $95^{\circ} \mathrm{C}$ and $60 \mathrm{sec}$ at $60^{\circ} \mathrm{C}$. Experiments were performed in triplicate using the $2^{-\Delta \Delta \mathrm{Cq}}$ method (23)

Transwell migration assay. The Transwell migration assay was performed using a 24-well Transwell chemotaxis chamber (EMD Millipore, Billerica, MA, USA). In brief, DMEM/F12 (500 $\mu \mathrm{l}$ ) supplemented with 2\% B-27, $10 \mathrm{ng} / \mathrm{ml} \mathrm{EGF,} \mathrm{and}$ $20 \mathrm{ng} / \mathrm{ml} \mathrm{bFGF}$ was placed in the lower chamber. A total of $2 \times 10^{4} \mathrm{CSCs}$ in a single-cell suspension in $200 \mu \mathrm{l}$ medium were 
seeded into the upper chamber (membrane pore size, $8 \mu \mathrm{m}$ ). The chamber was then incubated for $24 \mathrm{~h}$ at $37^{\circ} \mathrm{C}$ in a humidified atmosphere with $5 \% \mathrm{CO}_{2}$. The membrane was removed and cells on the upper surface which had not migrated were wiped away with a cotton swab. Subsequently, the membrane was fixed in $4 \%$ paraformaldehyde for $5 \mathrm{~min}$ at room temperature and then stained with $0.1 \%$ crystal violet (Sigma-Aldrich; Merck $\mathrm{KGaA}$ ) for $10 \mathrm{~min}$, followed by 3 washes with ice-cold PBS. The number of cells that had migrated to the lower surface of the membrane was counted in 10 random high-power fields under a light microscope (BL-AC10DS; Olympus, Tokyo, Japan). Each assay was performed in triplicate wells.

Serial replating experiments. Target cells were transfected with shHOTAIR or with LV-HOTAIR for $48 \mathrm{~h}$. For serial replating experiments, cells were replated at a clonal density (1,000 cells/well) and cultured in serum-free medium supplemented with $2 \%$ B-27, $10 \mathrm{ng} / \mathrm{ml} \mathrm{EGF}$ and $20 \mathrm{ng} / \mathrm{ml} \mathrm{bFGF}$. Every 3 days, the medium was half-replaced. After 14 days, cells were washed with PBS, fixed with $4 \%$ paraformaldehyde in PBS, stained with $0.1 \%$ crystal violet for $10 \mathrm{~min}$ and washed again with PBS, and the colonies were counted. For replating, the same amount of cells was plated in serum-free medium. After 14 days, the same procedure was performed three times.

Cell Counting kit-8 (CKK-8) proliferation assay. Cells at a concentration of $5 \times 10^{3}$ were seeded into 24 -well culture plates in $500 \mu \mathrm{l}$ culture medium supplemented with $2 \% \mathrm{~B}-27$, $10 \mathrm{ng} / \mathrm{ml}$ EGF and $20 \mathrm{ng} / \mathrm{ml} \mathrm{bFGF}$. Prior to detection, CCK-8 reagent (Sigma-Aldrich; Merck KGaA) was added at $10 \mu \mathrm{g} /$ well, followed by incubation for $2-4 \mathrm{~h}$ at $37^{\circ} \mathrm{C}$ and $5 \% \mathrm{CO}_{2}$ according to the manufacturer's protocols. A cell growth curve was drawn based on the corresponding normalized optical density values at $450 \mathrm{~nm}$ and each data-point represents the mean of three independent samples.

Flow cytometric analysis. For flow cytometric analysis of CSC markers, cells were detached using $0.25 \%$ Trypsin, re-suspended at $10^{6}$ cells $/ \mathrm{ml}$ and incubated with anti-CD24-fluorescein isothiocyanate (FITC; cat. no. FCMAB188F) and anti-CD44-phycoerythrin (cat.no. MABF582; EMD Millipore, Billerica, MA, USA) according to the manufacturer's protocols for $30 \mathrm{~min}$ on ice. Following washing with PBS three times, cells were fixed with $4 \%$ paraformaldehyde and then subjected to flow cytometric analysis.

Apoptosis was detected by flow cytometry following double staining with Annexin V-FITC and propidium iodide using the Annexin V-FITC Apoptosis Detection kit (BD BiosciencesFranklin Lakes, NJ, USA). A total of $0.5 \mathrm{ml}\left(1 \times 10^{6}\right.$ cells $\left./ \mathrm{ml}\right)$ of treated cells were washed in PBS, re-suspended in binding buffer supplied in the kit and stained with FITC-conjugated Annexin V (BD Pharmingen; BD Biosciences). After being stained for $30 \mathrm{~min}$ at $4^{\circ} \mathrm{C}$, the cells were incubated for $15 \mathrm{~min}$ in the dark at room temperature. Cells were re-washed with binding buffer and analysed using a flow cytometr (BD FACS Canto II; BD Biosciences).

Statistical analysis. Data were expressed as the mean \pm standard deviation. Multigroup comparisons of the mean were performed by one-way analysis and Specific contrasts were generated by Tukey's post hoc comparisons. using SPSS 16.0 software package (SPSS, Inc., Chicago, IL, USA. $\mathrm{P}<0.05$ was considered to indicate a statistically significant difference.

\section{Results}

Short-term exposure to gemcitabine induces expression of HOTAIR in PANC-1 CSCs. For detecting the expression profile of HOTAIR, MALAT-1, MEG3, PPP3CB, MAP3K14 and DAPK1 in PANC-1 and PANC-1 CSCs, CSCs were enriched from the PANC-1 population by incubation in serum-free medium. The self-renewal capacity of enriched CSCs was analyzed by a serial replating assay and the results confirmed their self-renewal capacity (Fig. 1A), while Panc-1 cells failed to form countable spheres because of its weak clonogenicity (data not shown). Detection of the population of CD24/CD $44^{+}$cells, which is the CSC population, also revealed a high enrichment compared with native PANC cells (Fig. 1B). The Panc-1 cells and the enriched Panc-1 CSCs with or without gemcitabine exposure were then subjected to RT-qPCR analysis. In Panc-1 cells, compared with untreated cells, $2 \mu \mathrm{M}$ gemcitabine exposure significantly upregulated MALAT-1 (Fig. 1C), and in Panc-1 CSCs, $2 \mu \mathrm{M}$ gemcitabine upregulated HOTAIR and MALAT-1 (Fig. 1D). As gemcitabine exposure did not affect HOTAIR in Panc-1 cells, the subsequent experiments focused on the regulatory roles of HOTAIR on Panc-1 CSCs.

Overexpression of HOTAIR increases chemoresistance to gemcitabine in PANC-1 CSCs. The upregulation of HOTAIR after gemcitabine treatment in CSCs prompted us to investigate the potential effects of HOTAIR on the chemoresistance of PANC-1 CSCs. PANC-1 CSCs were transfected by a HOTAIR-expressing lentivirus containing an GFP coding sequence for $48 \mathrm{~h}$. Subsequently, PANC-1 CSCs were imaged by fluorescent microscopy, revealing high efficiency of lentiviral transfection (Fig. 2A). For introducing HOTAIR into PANC-1 CSCs, lentivirus containing a coding sequence for HOTAIR (LV-HOTAIR) was packaged. At $48 \mathrm{~h}$ after transfection with LV-HOTAIR, the overexpression of HOTAIR compared with that in PANC-1 CSCs transfected with empty LV vector was confirmed by RT-qPCR (Fig. 2B). Subsequently, the sensitivity to gemcitabine was assessed, indicating that overexpression of HOTAIR significantly decreased the sensitivity of PANC-1 CSCs to gemcitabine (Fig. 2C). To further confirm the effect of HOTAIR on gemcitabine resistance, siHOTAIR was transfected into PANC-1 CSCs. Following knockdown of HOTAIR (Fig. 2B), the sensitivity of PANC-1 CSCs to gemcitabine was enhanced (Fig. 2E).

Overexpression of HOTAIR attenuates apoptosis and promotes proliferation of PANC-1 CSCs under gemcitabine treatment. According to the above results (Fig. 2), upregulation of HOTAIR in PANC-1 CSCs increased the concentration leading to $30 \%$ inhibition (IC30) and IC50 of gemcitabine. This prompted us to assess whether the resistance effect of HOTAIR attenuates apoptosis and promotes the proliferation of PANC-1 CSCs. After treatment with gemcitabine at the IC50 concentration for $24 \mathrm{~h}$, Annexin V/propidium iodide (PI) double labeling was performed for analyzing the apoptotic 
A

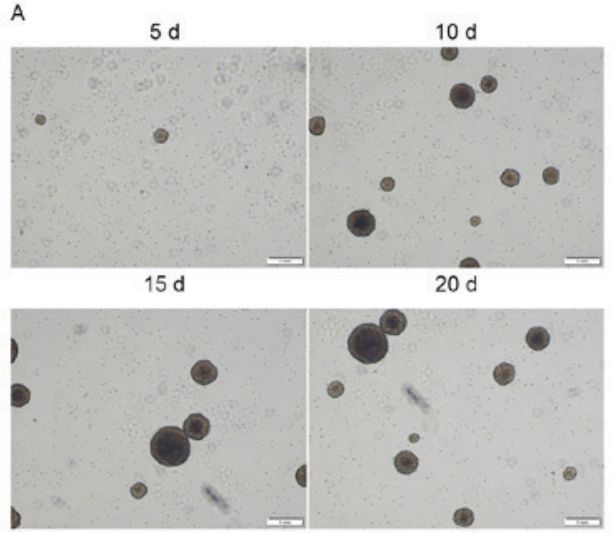

c
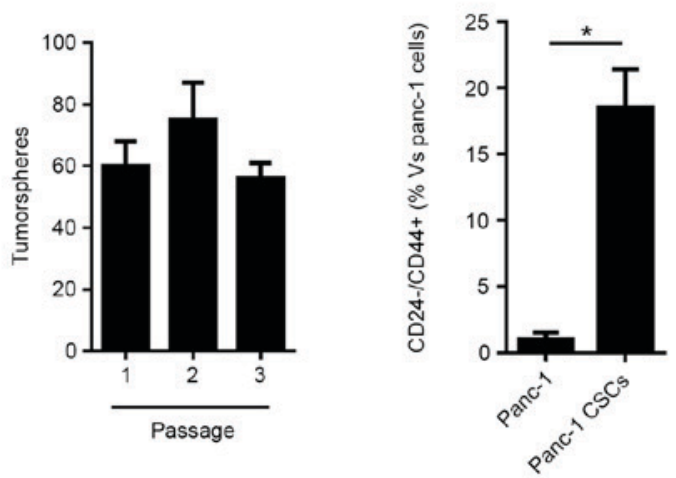
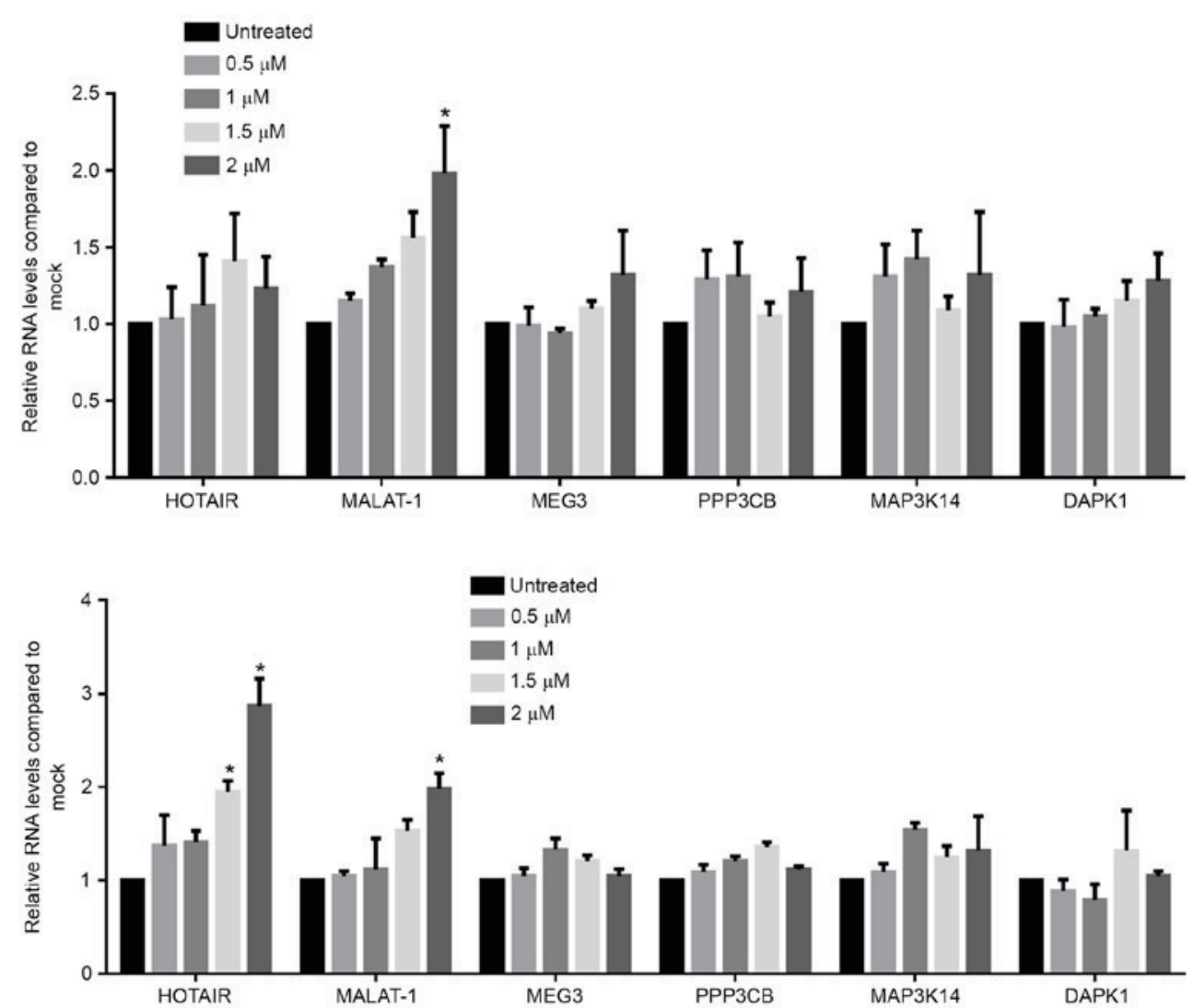

Figure 1. Gemcitabine exposure leads to upregulation of long non-coding RNA HOTAIR expression in Panc-1 CSCs. (A) Enrichment of CSCs from Panc-1 cells and identification of their self-renewal capacity by serial replating assay (scale bar, $1 \mathrm{~mm}$ ). (B) Flow cytometric analysis of CD24/CD44 ${ }^{+}$cells in Panc-1 cells and Panc-1 CSCs. The amount of CD24/CD44+ cells in Panc-1 cells was considered as 1. Reverse-transcription quantitative polymerase chain reaction analysis of the expression levels of HOTAIR, MALAT-1, MEG3, PPP3CB, MAP3K14 and DAPK1 in (C) Panc-1 cells or (D) Panc-1 CSCs with or without gemcitabine exposure. "P<0.05 vs. untreated or as indicated. CSCs, cancer stem-like cells; HOTAIR, HOX antisense intergenic RNA; MALAT-1, metastasis associated lung adenocarcinoma transcript 1; MEG3, maternally expressed 3; PPP3CB, protein phosphatase 3 catalytic subunit $\beta$; MAP3K14, mitogen-activated protein kinase kinase kinase 14; DAPK1, death-associated protein kinase 1; d, days.

rate. The results indicated that upregulation of HOTAIR, but not transfection with empty LV vector, decreased the ratio of early apoptotic cells (Annexin V-FITC ${ }^{+}$and PI $^{-}$due to intact cell membrane) and late apoptotic cells (Annexin $\mathrm{V}-F I T C^{+}$and $\mathrm{PI}^{+}$due to perforated cell membrane) (Fig. 3A). In comparison with untransfected cells, no detectable difference was observed in cells transfected with empty LV vector. Of note, the population of necrotic cells (Annexin $\mathrm{V}-\mathrm{FITC}-\mathrm{PI}^{+}$) exhibited a slight change (Fig. 3A). For detecting the effect of HOTAIR on the proliferation capacity, $1 \times 10^{4}$ transfected PANC-1 CSCs were incubated with the IC30 concentration of gemcitabine for
24-96 h, and the cellular viability was detected on each day. The results indicated that upregulation of HOTAIR promoted the proliferation of PANC-1 CSCs under gemcitabine treatment (Fig. 3B). Surprisingly, knockdown of HOTAIR by shHOTAIR introduction failed to significantly affect apoptosis or proliferation (data not shown), possibly due to the low expression levels of HOTAIR in unstressed PANC-1 CSCs.

Upregulation of HOTAIR affects the self-renewal capacity, migration and colony formation capacities of PANC-1 CSCs. The present study further investigated the regulatory roles of 
A
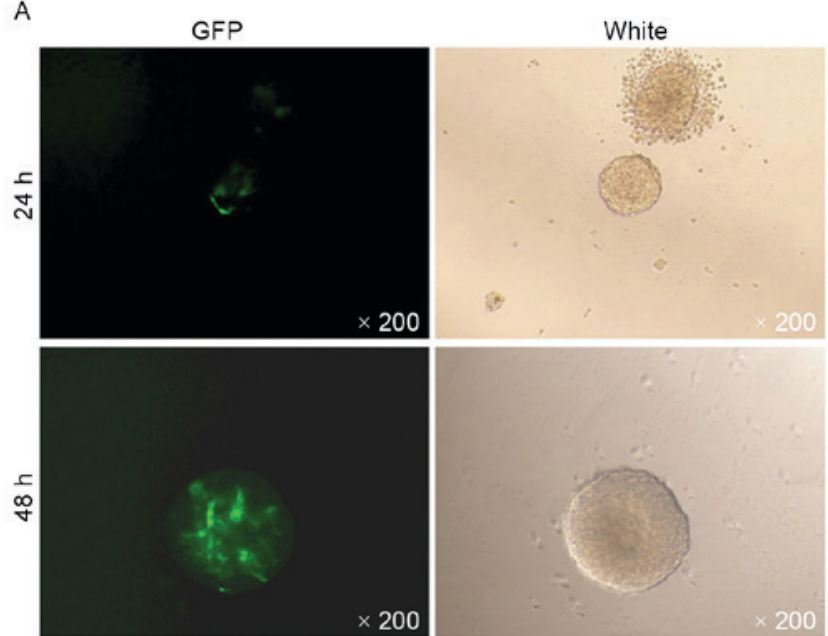

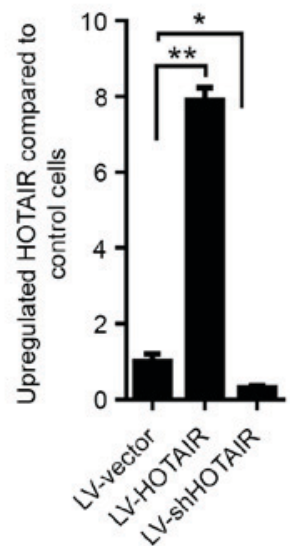

C

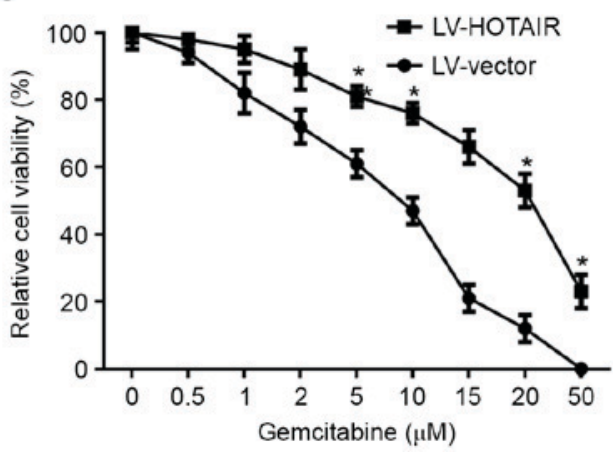

D

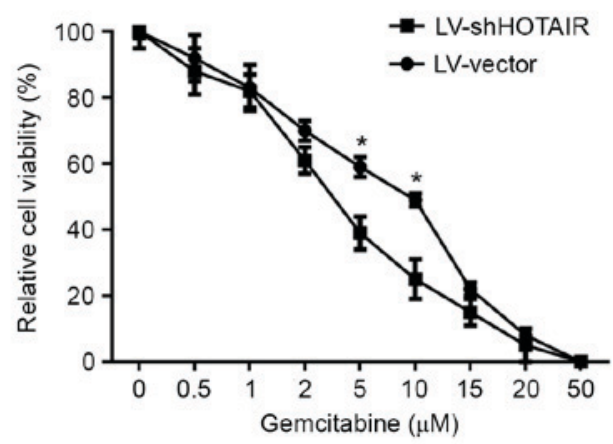

Figure 2. Overexpression of HOTAIR promotes chemoresistance to gemcitabine in Panc-1 CSCs. (A) The infectious efficiency was confirmed by fluorescent microscopy at $48 \mathrm{~h}$ after transfection (magnification, x200). (B) At $48 \mathrm{~h}$ after transfection, the expression levels of introduced HOTAIR were detected by reverse-transcription quantitative polymerase chain reaction analysis. (C) After HOTAIR overexpression and (D) after HOTAIR knockdown using shHOTAIR, the inhibitory effect of gemcitabine on the proliferation of Panc- 1 CSCs was measured using a Cell Counting kit- 8 assay. ${ }^{*} \mathrm{P}<0.05,{ }^{* *} \mathrm{P}<0.01$ vs. empty vector group. HOTAIR, HOX antisense intergenic RNA; CSCs, cancer stem-like cells; GFP, green fluorescence protein; LV, lentivirus; sh, small hairpin RNA.

upregulated HOTAIR in biological processes of PANC-1 CSCs. To analyse the impact of HOTAIR on the self-renewal capacity of PANC-1 CSCs, a well-established serial replating assay was used. LV-HOTAIR-transfected PANC-1 CSCs were able to form colonies in all four rounds of replating, and exhibited no significant difference in the initial three rounds of replating (Fig. 4A). However, the empty LV-vector-transfected PANC-1 CSCs exhibited a significant decrease in their self-renewal capacity during the 4th round of replating (Fig. 4A). A Transwell-based migration assay was established to quantitatively evaluate PANC-1 CSCs migration in vitro. As presented in Fig. 4B, compared with the control group, the average number of migrated PANC-1 CSCs increased significantly after HOTAIR introduction. To examine the effects of HOTAIR on colony formation in PANC-1 CSCs, a colony formation assay on soft agar was performed. The number of colonies formed by LV-HOTAIR-transfected PANC-1 CSCs was significantly increased compared with that of empty vector-transfected PANC-1 CSCs (Fig. 4C).

\section{Discussion}

The present study demonstrated that lncRNA HOTAIR was induced in Panc-1 CSCs after short-term gemcitabine exposure. Several lncRNAs tightly associated with malignancy of pancreatic cancer, including MALAT-1, HOTAIR, MEG3, PPP3CB, MAP3K14 and DAPK1 were detected in Panc-1 and Panc-1 CSCs, revealing that HOTAIR was investigated as its upregulation was CSC-specific. This prompted us to focus on the regulatory effects of HOTAIR induced by gemcitabine on the self-renewal capacity, proliferation, apoptosis and migration of Panc-1 CSCs. As expected, induction of HOTAIR by gemcitabine treatment promoted the proliferation and migration, maintained the self-renewal capacity and attenuated apoptosis of Panc-1 CSCs. Of note, following gemcitabine treatment for a relative long duration (96 h), HOTAIR expression was not significantly changed compared with that in untreated Panc-1 CSCs (data not shown). These results indicated that the induction of HOTAIR after gemcitabine exposure may be antagonized in an unknown manner. Taken together, induction of HOTAIR by short-term exposure to gemcitabine may contribute to the chemoresistance of Panc-1 CSCs. Furthermore, upregulation of HOTAIR led to the promotion of the proliferation and migration, maintenance of the self-renewal capacity and inhibition of apoptosis of Panc-1 CSCs after treatment with gemcitabine.

According to the CSC hypothesis, a small sub-population within a tumor has multipotent features and the capacity for indefinite self-renewal and asymmetric cell division $(24,25)$. Not 

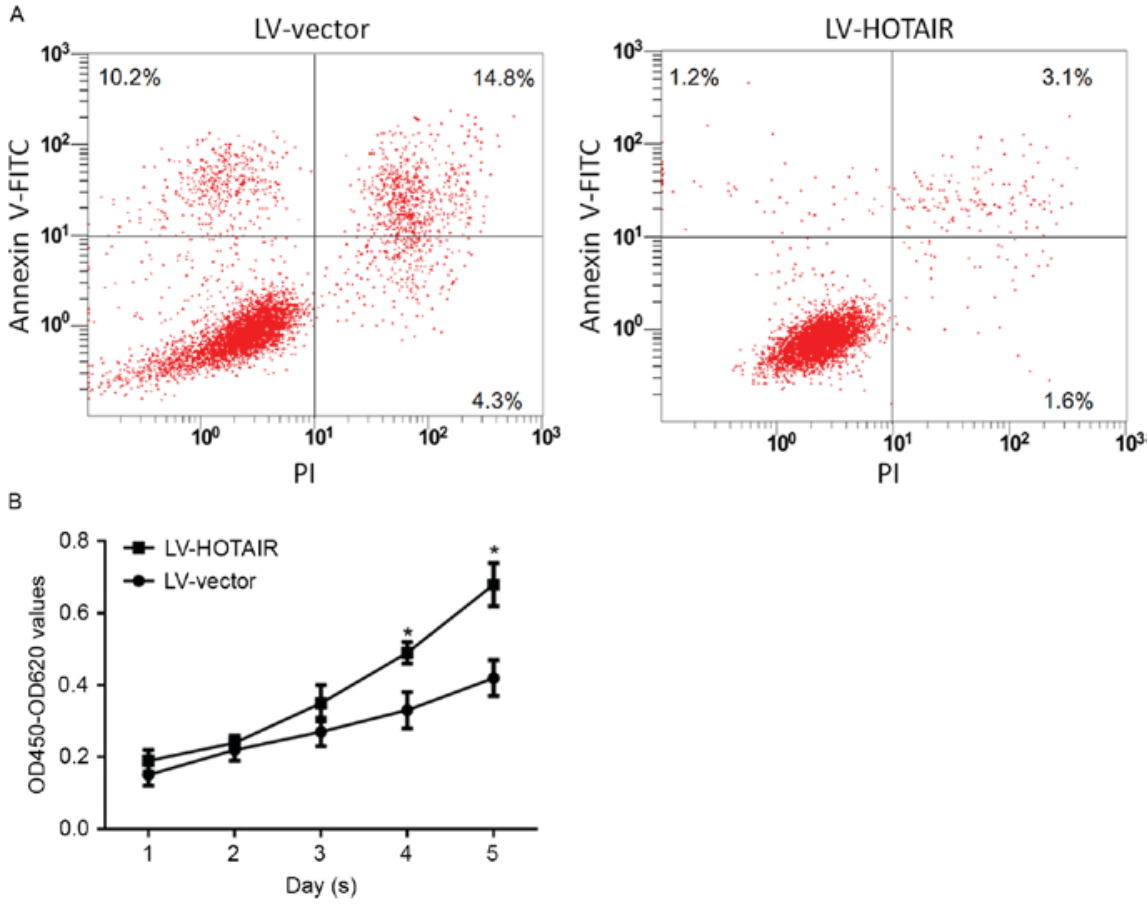

Figure 3. HOTAIR attenuates apoptosis and promotes the proliferation of Panc-1 CSCs after gemcitabine exposure. (A) Flow cytometric analysis was performed after Annexin V-FITC/PI double staining. (B) The proliferation of Panc-1 CSCs after gemcitabine exposure at the IC30 was assessed using a Cell Counting kit-8 assay. "P<0.05 vs. empty vector group. HOTAIR, HOX antisense intergenic RNA; CSCs, cancer stem-like cells; LV, lentivirus; OD, optical density; IC30, concentration leading to $30 \%$ inhibition; FITC, fluorescein isothiocyanate; PI, propidium iodide; OD, optical density.

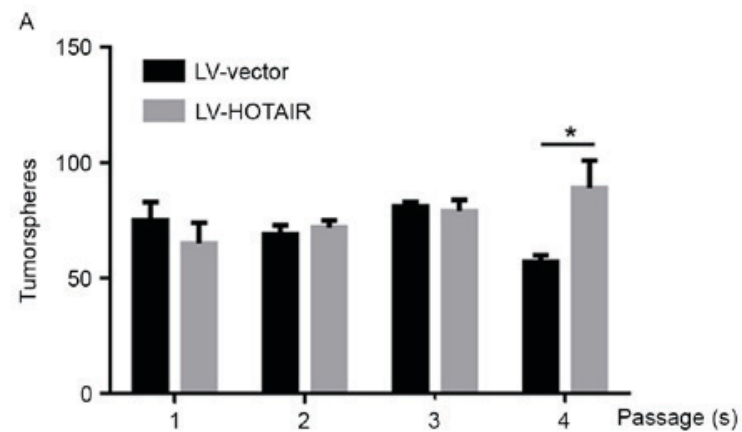

B
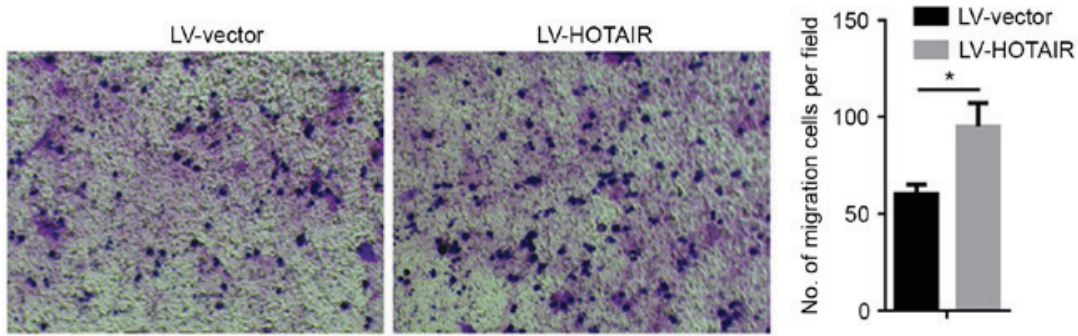

C
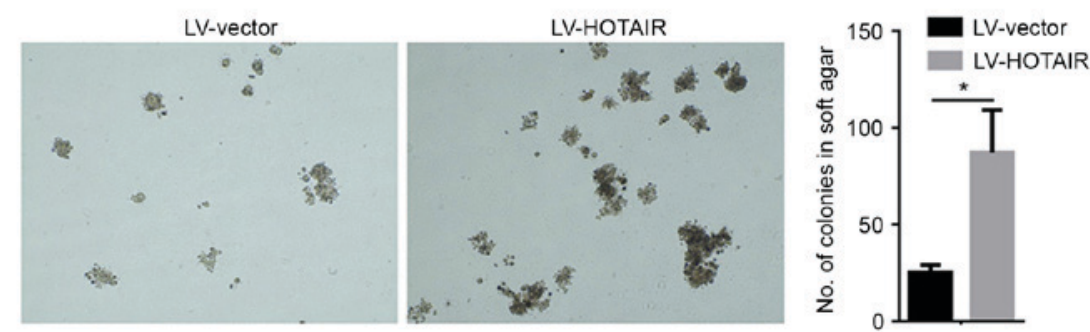

Figure 4. HOTAIR regulates the self-renewal, migration and colony formation capacity of Panc-1 CSCs. (A) Self-renewal capacity of LV-HOTAIR-transduced Panc-1 CSCs was assessed by a serial replating assay, (B) migration was assessed by a Transwell assay (magnification, $\mathrm{x} 100$ ) and (C) colony formation was assayed by replating in soft agar (magnification, $\mathrm{x} 100$ ). "P<0.05 vs. empty vector group. HOTAIR, HOX antisense intergenic RNA; CSCs, cancer stem-like cells; LV, lentivirus. 
only in carcinogenesis, accumulating evidence has indicated that CSCs may have a critical role in cancer aggressiveness, metastasis, recurrence and chemoresistance of solid tumors including pancreatic adenocarcinoma (26). CSCs were reported to be tightly associated with increased chemoresistance of pancreatic cancer with several mechanisms. Cioffi et al (27) found that, in pancreatic CSCs, downregulation of the microRNA-17-92 cluster promoted the self-renewal capacity as well as the in vivo tumorigenicity and chemoresistance by targeting multiple members of the Nodal/activin/transforming growth factor- $\beta 1$ signalling cascade. The enhanced efflux of Hoechst 33342 dye through adenosine triphosphate binding cassette transporters by CSCs demonstrated their chemoresistance mechanism through elimination of drug molecules (28). Furthermore, aldehyde dehydrogenase 1, a potential marker for CSCs, has been identified to have a potential role in chemoresistance (29). The role of B-cell lymphoma-2 (Bcl-2) protein and its family members has also been well explored as a novel mechanism of chemoresistance in CSCs (30). Collectively, CSCs of pancreatic cancer cells, contribute to chemoresistance via a variety of mechanisms.

The present study aimed to investigate the association and potential role of IncRNAs with the chemoresistant capacity of pancreatic CSCs, as emerging evidence has demonstrated the critical roles of lncRNAs in inducing chemoresistance in several cancer types. Li et al (31) reported that, in nasopharyngeal carcinoma (NPC), the recently identified lncRNA ROR is associated with the proliferation, metastasis, apoptosis and chemoresistance of NPC. MEG3 was revealed to be partially responsible for regulating cisplatin resistance of human lung adenocarcinoma cells through control of p53 and $\mathrm{Bcl}$ extra large protein expression (32). Of note, it was also reported that changes in the expression of ncRNAs may be associated with chemoresistance of non-small-cell lung cancer cells (33). As expected, HOTAIR was found to be induced by gemcitabine exposure and the ectopic expression of HOTAIR led to the promotion of proliferation and migration as well as maintenance of the self-renewal capacity of pancreatic CSCs.

In conclusion, the present study was the first, to the best of our knowledge, to demonstrate that IncRNA HOTAIR is induced by gemcitabine in pancreatic CSCs, and induction of HOTAIR expression led to promotion of proliferation and migration, maintenance of self-renewal capacity, attenuation of apoptosis and increase of chemoresistance. However, the exact mechanisms by which HOTAIR regulates these processes requires further elucidation. Based on these data, further study of the effects of HOTAIR on pancreatic CSCs is required in pathological tissues rather than a cell line. In addition, the regulation of associated genes and protein functions should also be studied. These further studies will help to improve the clinical treatment of pancreatic cancer.

\section{Acknowledgements}

The present study was supported by the Sichuan Provincial Scientific Grant (grant no. 2012SZ0141).

\section{References}

1. Siegel RL, Miller KD and Jemal A: Cancer statistics, 2015. CA Cancer J Clin 65: 5-29, 2015.
2. Li D, Xie K, Wolff R and Abbruzzese JL: Pancreatic cancer. Lancet 363: 1049-1057, 2004

3. O'Reilly EM and Abou-Alfa GK: Cytotoxic therapy for advanced pancreatic adenocarcinoma. Semin Oncol 34: 347-353, 2007.

4. Wang Z, Li Y, Ahmad A, Banerjee S, Azmi AS, Kong D and Sarkar FH: Pancreatic cancer: Understanding and overcoming chemoresistance. Nat Rev Gastroenterol Hepatol 8: 27-33, 2011.

5. Bergman AM, Pinedo HM and Peters GJ: Determinants of resistance to 2',2'-difluorodeoxycytidine (gemcitabine). Drug Resist Updat 5: 19-33, 2002.

6. Andersson R, Aho U, Nilsson BI, Peters GJ, Pastor-Anglada M, Rasch W and Sandvold ML: Gemcitabine chemoresistance in pancreatic cancer: Molecular mechanisms and potential solutions. Scand J Gastroenterol 44: 782-786, 2009.

7. Polyak K and Hahn WC: Roots and stems: Stem cells in cancer. Nat Med 12: 296-300, 2006.

8. Kakarala M and Wicha MS: Implications of the cancer stem-cell hypothesis for breast cancer prevention and therapy. J Clin Oncol 26: 2813-2820, 2008.

9. Singh SK, Clarke ID, Terasaki M, Bonn VE, Hawkins C, Squire J and Dirks PB: Identification of a cancer stem cell in human brain tumors. Cancer Res 63: 5821-5828, 2003.

10. Abdullah LN and Chow EK: Mechanisms of chemoresistance in cancer stem cells. Clin Transl Med 2: 3, 2013.

11. Ucar D, Cogle CR, Zucali JR, Ostmark B, Scott EW, Zori R, Gray BA and Moreb JS: Aldehyde dehydrogenase activity as a functional marker for lung cancer. Chem Biol Interact 178: 48-55, 2009.

12. Jimeno A, Feldmann G, Suárez-Gauthier A, Rasheed Z, Solomon A, Zou GM, Rubio-Viqueira B, García-García E, López-Ríos F, Matsui W, et al: A direct pancreatic cancer xenograft model as a platform for cancer stem cell therapeutic development. Mol Cancer Ther 8: 310-314, 2009.

13. Hellsten R, Johansson M, Dahlman A, Sterner O and Bjartell A: Galiellalactone inhibits stem cell-like ALDH-positive prostate cancer cells. PLoS One 6: e22118, 2011.

14. Ma S, Chan KW, Lee TK, Tang KH, Wo JY, Zheng BJ and Guan XY: Aldehyde dehydrogenase discriminates the CD133 liver cancer stem cell populations. Mol Cancer Res 6: 1146-1153, 2008.

15. Clay MR, Tabor M, Owen JH, Carey TE, Bradford CR, Wolf GT, Wicha MS and Prince ME: Single-marker identification of head and neck squamous cell carcinoma cancer stem cells with aldehyde dehydrogenase. Head Neck 32: 1195-1201, 2010.

16. Yin T, Wei H, Gou S, Shi P, Yang Z, Zhao G and Wang C: Cancer stem-like cells enriched in Panc-1 spheres possess increased migration ability and resistance to gemcitabine. Int J Mol Sci 12: 1595-1604, 2011

17. Yang G, Lu X and Yuan L: LncRNA: A link between RNA and cancer. Biochim Biophys Acta 1839: 1097-1109, 2014.

18. Jiao F, Hu H, Han T, Yuan C, Wang L, Jin Z, Guo Z and Wang L: Long noncoding RNA MALAT-1 enhances stem cell-like phenotypes in pancreatic cancer cells. Int J Mol Sci 16: 6677-6693, 2015.

19. Zhuang Y, Wang X, Nguyen HT, Zhuo Y, Cui X, Fewell C, Flemington EK and Shan B: Induction of long intergenic non-coding RNA HOTAIR in lung cancer cells by type I collagen. J Hematol Oncol 6: 35, 2013.

20. Zhang A, Zhao JC, Kim J, Fong KW, Yang YA, Chakravarti D, Mo YY and Yu J: LncRNA HOTAIR enhances the androgen-receptor-mediated transcriptional program and drives castration-resistant prostate cancer. Cell Rep 13: 209-221, 2015.

21. Kim K, Jutooru I, Chadalapaka G, Johnson G, Frank J, Burghardt R, Kim S and Safe S: HOTAIR is a negative prognostic factor and exhibits pro-oncogenic activity in pancreatic cancer. Oncogene 32: 1616-1625, 2013.

22. Tahira AC, Kubrusly MS, Faria MF, Dazzani B, Fonseca RS, Maracaja-Coutinho V, Verjovski-Almeida S, Machado MC and Reis EM: Long noncoding intronic RNAs are differentially expressed in primary and metastatic pancreatic cancer. Mol Cancer 10: 141, 2011.

23. Livak KJ and Schmittgen TD: Analysis of relative gene expression data using real-time quantitative PCR and the 2(-Delta Delta C(T)) method. Methods 25: 402-408, 2001.

24. Lee CJ, Dosch J and Simeone DM: Pancreatic cancer stem cells. J Clin Oncol 26: 2806-2812, 2008.

25. Hermann PC, Mueller MT and Heeschen C: Pancreatic cancer stem cells-insights and perspectives. Expert Opin Biol Ther 9: 1271-1278, 2009 
26. Habib M and Saif MW: Pancreatic cancer stem cells: Their role in pancreatic cancer patient outcomes and what is future? JOP 14: 401-404, 2013.

27. Cioffi M, Trabulo SM, Sanchez-Ripoll Y, Miranda-Lorenzo I, Lonarod E, Dorado J, Reis Vieira C, Ramirez JC, Hidalgo M, Aicher A, et al: The miR-17-92 cluster counteracts quiescence and chemoresistance in a distinct subpopulation of pancreatic cancer stem cell. Gut 64: 1936-1948, 2015.

28. Goodell MA, Brose K, Paradis G, Conner AS and Mulligan RC: Isolation and functional properties of murine hematopoietic stem cells that are replicating in vivo. J Exp Med 183: 1797-1806, 1996.

29. Hilton J: Role of aldehyde dehydrogenase in cyclophosphamide-resistant L1210 leukemia. Cancer Res 44: 5156-5160, 1984

30. Kim R, Emi M and Tanabe K: Role of mitochondria as the gardens of cell death. Cancer Chemother Pharmacol 57: 545-553, 2006.
31. Li L, Gu M, You B, Shi S, Shan Y, Bao L and You Y: Long non-coding RNA ROR promotes proliferation, migration and chemoresistance of nasopharyngeal carcinoma. Cancer Sci 107: 1215-1222, 2016

32. Liu J, Wan L, Lu K, Sun M, Pan X, Zhang P, Lu B, Liu G and Wang Z: The long noncoding RNA MEG3 contributes to cisplatin resistance of human lung adenocarcinoma. PLoS One 10: e0114586, 2015.

33. Yang Y, Li H, Hou S, Hu B, Liu J and Wang J: The noncoding RNA expression profile and the effect of lncRNAAK126698 on cisplatin resistance in non-small-cell lung cancer cell. PLoS One 8: e65309, 2013. 